\title{
Use of aspirin in Chinese after recovery from primary intracranial haemorrhage
}

Boon-Hor Chong'; Koon-Ho Chan²; Vincent Pong1; Kui-Kai Lau²; Yap-Hang Chan'; Ming-Liang Zuo'; Wai-Man Lui³; Gilberto Ka-Kit Leung ${ }^{3}$; Chu-Pak Lau'; Hung-Fat Tse'; Jenny Kan-Suen Pu33; Chung-Wah Siu ${ }^{1}$

${ }^{1}$ Cardiology Division, Department of Medicine, Li Ka Shing Faculty of Medicine, the University of Hong Kong, Hong Kong SAR, China; ${ }^{2}$ Neurology Division, Department of Medicine, Li Ka Shing Faculty of Medicine, the University of Hong Kong, Hong Kong SAR, China; ${ }^{3}$ Department of Neurosurgery, Li Ka Shing Faculty of Medicine, the University of Hong Kong, Hong Kong SAR, China; ${ }^{4}$ Department of Community Medicine, Li Ka Shing Faculty of Medicine, the University of Hong Kong, Hong Kong SAR, China

\begin{abstract}
Summary
Intracranial haemorrhage (ICH) accounts for $\sim 35 \%$ of all strokes in Chinese. Anti-platelet agent is often avoided after an index event due to the possibility of recurrent ICH. This single-centered observational study included 440 consecutive Chinese patients with a first spontaneous ICH surviving the first month performed during 1996-2010. The subjects were identified, and their clinical characteristics, anti-platelet therapy after ICH, and outcomes including recurrent ICH, ischaemic stroke, and acute coronary syndrome were checked from hospital records. Of these 440 patients, 56 patients $(12.7 \%)$ were prescribed aspirin (312 patientaspirin years). After a follow-up of $62.2 \pm 1.8$ months, 47 patients had recurrent ICH (10.7\%, 20.6 per 1,000 patient years). Patients prescribed aspirin did not have a higher risk of recurrent ICH compared with those not prescribed aspirin (22.7 per 1,000 patient-aspirin years vs. 22.4 per 1,000 patient years, $p=0.70$ ). Multivariate analysis identified age $>60$
\end{abstract}

years (hazard ratio $[\mathrm{HR}]: 2.0,95 \%$ confidence interval $[\mathrm{Cl}]: 1.07-3.85$, $\mathrm{p}=0.03$ ) and hypertension (HR: 2.0,95\% Cl: 1.06-3.75, $\mathrm{p}=0.03$ ) as independent predictors for recurrent ICH. In a subgroup analysis including 127 patients with standard indications for aspirin of whom 56 were prescribed aspirin, the incidence of combined vascular events including recurrent ICH, ischaemic stroke, and acute coronary syndrome was statistically lower in patients prescribed aspirin than those not prescribed aspirin (52.4 per 1,000 patient-aspirin years, vs. 112.8 per 1,000 patient-years, $p=0.04)$. In conclusion, we observed in a cohort of Chinese post-ICH patients that aspirin use was not associated with an increased risk for a recurrent ICH.

\section{Keywords}

Antiplatelet agents, risk factors, stroke / prevention
Correspondence to:

Chung Wah Siu, MD or Jenny Kan-Suen Pu, MBBS

Cardiology Division, Department of Medicine

The University of Hong Kong, Queen Mary Hospital

Hong Kong, China

Tel.: +852 2855 3600, Fax: +852 28186304

E-mail: cwdsiu@hkucc.hku.hk or ks.jpu@hkucc.hku.hk
Received: June 29, 2011

Accepted after major revision: November 4, 2011

Prepublished online: December 21, 2011

doi:10.1160/TH11-06-0439

Thromb Haemost 2012; 107: 241-247

\section{Introduction}

Spontaneous intracranial haemorrhage (ICH) accounts for $10-15 \%$ of all strokes in Caucasians (1-3) and up to $35 \%$ in Chinese (4-6), and results in significant morbidity and mortality. Approximately $50 \%$ of patients with ICH die within 30 days of the index haemorrhage, and of those who survive, only a minority are expected to be functionally independent (7). Those who survive the initial event also face a substantial risk of recurrent ICH. Many risk factors for $\mathrm{ICH}$, particularly advanced age, hypertension and previous ischaemic stroke, are also risk factors for atherosclerotic disease. Thus individuals who survive an $\mathrm{ICH}$ are also at risk of other future thrombotic events such as ischaemic stroke. A previous long-term follow-up study demonstrated substantial risk among ICH survivors for ischaemic stroke, myocardial infarction, and sudden death, in addition to recurrent ICH (8). Despite the high thrombotic risk, clinicians may be reluctant to prescribe antiplatelet therapy to post-ICH patients that may consequently in- crease the risk of recurrent ICH (9). The benefits of long-term anti-platelet therapy must be weighed against the risk of recurrent $\mathrm{ICH}$; clinical data of such treatment in patients following $\mathrm{ICH}$ is nonetheless limited. Recent evidence has revealed an association between anti-platelet therapy and increased risk of recurrent ICH in Caucasians $(10,11)$. It remains unclear whether the same applies in post-ICH Chinese patients. This study aimed to investigate the clinical outcome of post-ICH patients prescribed antiplatelet therapy using a single-centre registry database.

\section{Methods}

\section{Study population}

We recruited 691 consecutive patients (mean age 62.1 \pm 0.6 years, male $60.1 \%$ ) who were admitted to the neurosurgical ward, of 
Queen Mary Hospital, Hong Kong, China with a first spontaneous ICH, from May 1996 to February 2010. Located at the western region of Hong Kong Island, Queen Mary Hospital is the largest hospital of the area and serves a population of about half a million. Patients were excluded if they were under 18 years of age, had ICH secondary to trauma, arteriovenous malformation, aneurysm, moya-moya disease, haemorrhagic transformation of an ischaemic stroke or complication following other neurosurgical intervention, and/or died within 30 days of first ICH. Patients prescribed warfarin during follow-up were also excluded from the analysis. A total of 440 patients (mean age $59.2 \pm 0.8$ years, male: $62.3 \%$ ) were included in the final analysis ( Fig. 1).

\section{Study design}

This was a single-centre registry based on the clinical outcomes of Chinese patients with first spontaneous ICH. After admission, data pertaining to the index ICH, demographics, cardiovascular risk factors and medication, particularly previous use of anti-thrombotic medication (aspirin, clopidogrel, and warfarin), were entered into the Clinical Management System Database of the hospital. ICHs were classified into intra-cerebral haemorrhage, subarachnoid haemorrhage, and subdural haemorrhage. The location of intra-cerebral haemorrhage was classified as lobar (grey matter and the underlying subcortical white matter) and deep cerebral (periventricular white matter, caudate, globus pallidus, putamen, internal capsule, thalamus, cerebellar, and brainstem) (11). For cases designated as pure intraventricular haemorrhage after review of all available films, they were categorised as deep intra-cerebral haemorrhage. After discharge, all patients were followed-up in our outpatient clinic. Clinical data concerning the subsequent use of anti-thrombotic therapy, recurrent ICH, major adverse cardiovascular events and death during the follow-up period were retrieved from medical records and discharge summaries from all local hospitals. Patients who were lost to follow-up were contacted by phone. Survival data were also obtained from the Births and Deaths General Register Office.

\section{Endpoints and definitions}

The major clinical endpoints were recurrence of $\mathrm{ICH}$, ischaemic stroke and acute coronary syndrome. ICH was diagnosed in the presence of new onset neurological symptoms with radiological confirmation (computerised axial tomography [CAT] scan or magnetic resonance imaging [MRI]), and classified as intra-cerebral haemorrhage, subarachnoid haemorrhage or subdural haemorrhage (12). Ischaemic stroke was defined as a neurological deficit of sudden onset that persisted for more than 24 hours, and that corresponded to a vascular territory in the absence of primary haemorrhage or other cause (trauma, infection, vasculitis) and confirmed by CAT scan or MRI of the brain (12-14). Acute coron- ary syndrome was defined as the triad of acute ischaemic symptoms (lasting 30 minutes or longer) at rest, typical electrocardiographic changes and/or positive cardiac markers (elevated cardiac troponins or creatine kinase $>2$ times the upper limit of normal range) (15-17).

\section{Statistical analysis}

Continuous variables were expressed as the mean \pm standard error of mean (SEM). Statistical comparisons were performed using the Student t-test or Fisher exact test, as appropriate. Event rates were calculated as the number of events divided by patient-years of exposure to aspirin. Hazard ratios (HR) and 95\% confidence intervals (CI) were calculated by univariate and multivariate Cox proportional hazards regression models. Multivariate analyses were performed with an enter regression model in which each variable with a p-value of $\leq 0.1$ (based on the univariate analysis) was entered into the model. Calculations were performed using a statistical software package (SPSS, version 12.0; SPSS; Chicago, IL, USA). A p-value $<0.05$ was considered to be statistically significant.

\section{Results}

During the study period, 691 consecutive patients were admitted for a first-time ICH. Among these patients, 48 with traumatic causes of ICH, 62 with arteriovenous malformation, 22 with aneurysm, 11 with moya-moya disease, 10 with haemorrhagic transformation of an ischaemic stroke, 75 died within 30 days of first $\mathrm{ICH}$, and 23 prescribed warfarin after the first ICH were thus excluded. As a result, a total of 440 who fulfilled the study entry criteria were included in analysis ( Fig. 1). Table 1 summarises the clinical characteristics of the study population. The mean age was $59.2 \pm 0.8$ years with a male predominance of $62.3 \%$. Intra-cerebral haemorrhage accounted for the majority (93.6\%) of all ICHs, with subarachnoid haemorrhage (5.2\%) and subdural haemorrhage (1.2\%) making up the remainder. Anti-thrombotic medications were taken by $17.3 \%$ of all patients (aspirin: $14.1 \%$, warfarin: $2.7 \%$, and clopidogrel: $0.5 \%$ ) prior to the index ICH. Surgical evacuation of the hematoma was performed in 214 patients (48.6\%).

\section{Follow-up}

The mean follow-up duration was $62.2 \pm 1.8$ months (a total of 2,281 patient-years follow-up). No patients were lost in follow-up. Aspirin was subsequently prescribed to 56 of the 440 patients $(12.7 \%)$ during this period. All patients prescribed aspirin were at dosage of $100 \mathrm{mg}$ daily. The mean time from ICH to the prescription of aspirin was $27.8 \pm 4.1$ months and mean duration of aspirin exposure was $28.3 \pm 3.5$ months (132 patient-aspirin-years). 
Table 2 summarises the baseline characteristics of patients prescribed aspirin and not prescribed aspirin after ICH. As expected, a higher proportion of patients prescribed aspirin after ICH had cardiovascular risk factors including diabetes (28.6\% vs. $13.8 \%$, $\mathrm{p}<0.01)$, hypertension ( $67.9 \%$ vs. $49.7 \%, \mathrm{p}=0.01)$, coronary artery disease (32.1\% vs. $2.9 \%, \mathrm{p}<0.01)$, congestive heart failure ( $8.9 \%$ vs. $1.6 \%, \mathrm{p}<0.01)$, previous stroke and/or transient ischaemic attack ( $19.6 \%$ vs. $7.3 \%, \mathrm{p}<0.01)$, and atrial fibrillation ( $16.1 \%$ vs. $2.9 \%$, $\mathrm{p}<0.01)$ compared with patients not prescribed aspirin.

\section{Recurrent ICH}

Recurrent ICH was observed in 47 patients $(10.7 \%, 20.6$ per 1,000 patient years) of whom 44 were not prescribed aspirin (92.9\%) and three were $(7.1 \%)$. The incidence of recurrent ICH in patients not prescribed aspirin was 22.4 per 1,000 patient years, which was not significantly different to patients prescribed aspirin (22.7 per 1,000 patient-aspirin years, $\mathrm{p}=0.70)$. In Cox regression analysis, the only predictors for recurrent ICH were age $>60$ years (HR: 2.0, 95\% CI: $1.07-3.85, \mathrm{p}=0.03$ ) and hypertension (HR: 2.0, 95\% CI: 1.06-3.75, $\mathrm{p}=0.03$ ). Use of aspirin after ICH was not associated with recurrent $\mathrm{ICH}$.

In a subgroup analysis, we further subdivided 412 patients with intra-cerebral haemorrhage according to the location of haemorrhage: 142 patients (34.4\%) with lobar haemorrhage and 270 patients $(65.6 \%)$ with deep cerebral haemorrhage. Among patients with lobar haemorrhage, 20 patients were prescribed aspirin after the first ICH. During the follow-up period, altogether 17 patients developed recurrent ICH (21.9 per 1,000 patient years in patients with lobar haemorrhage), but none of them were prescribed aspirin after the first ICH. The incidence of recurrent ICH of patients not prescribed aspirin was 25.1 per 1,000 patient years, which was
Table 1: Baseline characteristics of patients with ICH surviving the first 30 days.

\begin{tabular}{|l|l|}
\hline & $\begin{array}{l}\text { First-30-day } \\
\text { survivors }\end{array}$ \\
\hline Number & 440 \\
\hline Male, $\mathrm{n}(\%)$ & $274(62.3)$ \\
\hline Age (years) & $59.3 \pm 0.8$ \\
\hline Diabetes, $\mathrm{n}(\%)$ & $69(15.6)$ \\
\hline Hypertension, $\mathrm{n}(\%)$ & $229(52.0)$ \\
\hline Smoking history, $\mathrm{n}(\%)$ & $116(26.4)$ \\
\hline Coronary artery disease, $\mathrm{n}(\%)$ & $29(6.6)$ \\
\hline Congestive heart failure, $\mathrm{n}(\%)$ & $11(2.5)$ \\
\hline Previous stroke/transient ischaemic attack, $\mathrm{n}(\%)$ & $39(8.9)$ \\
\hline Atrial fibrillation, $\mathrm{n}(\%)$ & $20(4.5)$ \\
\hline Types of intracranial haemorrhage & \\
\hline Intracerebral haemorrhage, $\mathrm{n}(\%)$ & $412(93.6)$ \\
\hline Subarachnoid haemorrhage, $\mathrm{n}(\%)$ & $23(5.2)$ \\
\hline Subdural haemorrhage, $\mathrm{n}(\%)$ & $5(1.2)$ \\
\hline Evacuation of haematoma, $\mathrm{n}(\%)$ & $214(48.6)$ \\
\hline Anti-thrombotic therapy prior to ICH & \\
\hline Warfarin, $\mathrm{n}(\%)$ & $12(2.7)$ \\
\hline Aspirin, $\mathrm{n}(\%)$ & $62(14.1)$ \\
\hline Clopidogrel, $\mathrm{n}(\%)$ & $2(0.5)$ \\
\hline & \\
\hline
\end{tabular}

not statistically different from patients prescribed aspirin $(\mathrm{p}=0.30)$. For patients with deep cerebral haemorrhage, 32 out of 270 patients were prescribed aspirin after the first ICH. There were 25 patients developed recurrent ICH: 22 in patients not prescribed aspirin $(9.2 \%)$ and three in patients prescribed aspirin (9.4\%). The
Table 2: Characteristics of patients with and without aspirin.

\begin{tabular}{|l|l|l|l}
\hline & No aspirin & Aspirin & P-value \\
\hline Number & 384 & 56 & \\
\hline Male, $\mathrm{n}(\%)$ & $236(61.5)$ & $38(67.8)$ & 0.36 \\
\hline Age (years) & $58.5 \pm 0.8$ & $64.2 \pm 1.7$ & $0.01^{*}$ \\
\hline Diabetes, $\mathrm{n}(\%)$ & $53(13.8)$ & $16(28.6)$ & $<0.01^{*}$ \\
\hline Hypertension, $\mathrm{n}(\%)$ & $191(49.7)$ & $38(67.9)$ & $0.01^{*}$ \\
\hline Smoking history, $\mathrm{n}(\%)$ & $97(25.3)$ & $19(33.9)$ & 0.17 \\
\hline Coronary artery disease, $\mathrm{n}(\%)$ & $11(2.9)$ & $18(32.1)$ & $<0.01^{*}$ \\
\hline Congestive heart failure, $\mathrm{n}(\%)$ & $6(1.6)$ & $5(8.9)$ & $<0.01^{*}$ \\
\hline Previous stroke/transient ischaemic attack, $\mathrm{n}(\%)$ & $28(7.3)$ & $11(19.6)$ & $<0.01^{*}$ \\
\hline Atrial fibrillation, $\mathrm{n}(\%)$ & $11(2.9)$ & $9(16.1)$ & $<0.01^{*}$ \\
\hline Types of intracranial haemorrhage & & & 0.53 \\
\hline Intracerebral haemorrhage, $\mathrm{n}(\%)$ & $360(93.8)$ & $52(92.9)$ & \\
\hline Subarachnoid haemorrhage, $\mathrm{n}(\%)$ & $19(4.9)$ & $4(7.1)$ & \\
\hline Subdural haemorrhage, $\mathrm{n}(\%)$ & $5(1.3)$ & $0(0)$ & \\
\hline Evacuation of haematoma, $\mathrm{n}(\%)$ & $191(49.7)$ & $23(41.1)$ & 0.23 \\
\hline
\end{tabular}




\begin{tabular}{|l|l|l|l}
\hline & No aspirin & Aspirin & P-value \\
\hline Number & 71 & 56 & \\
\hline Male, $\mathrm{n}(\%)$ & $42(59.2)$ & $38(67.8)$ & 0.31 \\
\hline Age (years) & $65.5 \pm 1.6$ & $64.2 \pm 1.7$ & 0.58 \\
\hline Diabetes, $\mathrm{n}(\%)$ & $53(74.6)$ & $16(28.6)$ & $<0.01^{*}$ \\
\hline Hypertension, $\mathrm{n}(\%)$ & $60(84.5)$ & $38(67.9)$ & $0.03^{*}$ \\
\hline Smoking history, $\mathrm{n}(\%)$ & $27(38.0)$ & $19(33.9)$ & 0.63 \\
\hline Coronary artery disease, $\mathrm{n}(\%)$ & $11(15.5)$ & $18(32.1)$ & $0.03^{*}$ \\
\hline Congestive heart failure, $\mathrm{n}(\%)$ & $4(5.6)$ & $5(8.9)$ & 0.51 \\
\hline Previous stroke/transient ischaemic attack, $\mathrm{n}(\%)$ & $14(19.7)$ & $11(19.6)$ & 0.99 \\
\hline Atrial fibrillation, $\mathrm{n}(\%)$ & $11(15.5)$ & $9(16.1)$ & 0.93 \\
\hline Types of intracranial haemorrhage & & & 0.36 \\
\hline Intracerebral haemorrhage, $\mathrm{n}(\%)$ & $66(93.0)$ & $52(92.9)$ & \\
\hline Subarachnoid haemorrhage, $\mathrm{n}(\%)$ & $3(4.2)$ & $4(7.1)$ & \\
\hline Subdural haemorrhage, $\mathrm{n}(\%)$ & $2(2.8)$ & $0(0)$ & \\
\hline Evacuation of haematoma, $\mathrm{n}(\%)$ & $33(46.5)$ & $23(41.1)$ & 0.54 \\
\hline
\end{tabular}

Table 3: Characteristics of high-risk patients with and without aspirin. overall incidence of recurrent ICH in patients with deep cerebral haemorrhage was 18.2 per 1,000 patient years. Although the incidence of recurrent ICH in patients prescribed aspirin during follow-up period was higher than those not prescribed aspirin (38.7 per 1,000 patient-aspirin years vs. 20.8 per 1,000 patient years), this difference was not statistically significant $(\mathrm{p}=0.11)$. Due to small sample size and low event rate in these sub-groups, it was not possible to perform Cox-regression analysis.

\section{Ischaemic stroke and acute coronary syndrome}

Ischaemic stroke occurred in 29 patients (12.7 per 1,000 patient years) during the follow-up period: 24 occurred in patients not prescribed aspirin, and five in patients prescribed aspirin. The incidence of ischaemic stroke in patients prescribed aspirin after ICH was 44.4 per 1,000 patient-aspirin years, significantly higher than in those not prescribed aspirin (12.7 per 1,000 patient years, $\mathrm{p}=0.03$ ). Further, in patients with lobar haemorrhage, the incidence of ischaemic stroke in patients prescribed aspirin after ICH was 57.2 per 1,000 patient-aspirin years compared with 7.4 per $1,000$ patient years in those not prescribed aspirin ( $\mathrm{p}=0.06)$. In addition, in patients with deep cerebral haemorrhage, the incidence of ischaemic stroke in patients prescribed aspirin after ICH was 154.9 per 1,000 patient-aspirin years, significantly higher than in those not prescribed aspirin (6.7 per 1,000 patient years, $\mathrm{p}<0.01)$.

In addition, there were also 25 incidences of acute coronary syndrome observed during the follow-up period: 13 occurred in patients not prescribed aspirin and 12 in patients prescribed aspirin. The incidence of acute coronary syndrome in patients prescribed aspirin after ICH and those not prescribed aspirin was 6.9 per 1,000 patient-aspirin years and 92.3 per 1,000 patient years $(\mathrm{p}<0.01)$, respectively. In subgroup analysis according to the lo- cation of haemorrhage, the incidence of acute coronary syndrome in patients with lobar haemorrhage and prescribed aspirin after ICH was 4.4 per 1,000 patient-aspirin years compared with 133.4 per 1,000 patient years in those not prescribed aspirin $(\mathrm{p}<0.01)$. Among patients with deep cerebral haemorrhage, the incidence of acute coronary syndrome in patients prescribed aspirin after ICH was 64.5 per 1,000 patient-aspirin years, significantly higher than in those not prescribed aspirin (5.8 per 1,000 patient years, $\mathrm{p}<0.01)$.

\section{Outcome of high-risk patients}

The higher incidence of ischaemic stroke and acute coronary syndrome in patients prescribed aspirin following ICH is related to the higher prevalence of underlying thrombotic disease and/or other cardiovascular risk factors. In a subgroup analysis, only patients with standard clinical indications for anti-platelet therapy, namely coronary artery disease, ischaemic stroke, atrial fibrillation, and diabetes mellitus, were included: 127 patients were included in this subgroup analysis of whom 56 were prescribed aspirin. Baseline characteristics according to aspirin use are summarised in Table 3. There were no significant differences in age, sex, smoking history, history of ischaemic stroke and/or transient ischaemic attack, and atrial fibrillation between the two groups; nonetheless patients prescribed aspirin following ICH had a higher prevalence of coronary artery disease $(32.1 \%$ vs. $15.5 \%, \mathrm{p}=0.03)$, but lower prevalence of hypertension $(67.9 \%$ vs. $84.5 \%, \mathrm{p}=0.03)$ and diabetes $(28.6 \%$ vs. $74.6 \%, p<0.01)$ compared with patients not prescribed aspirin. There were no significant differences in types of ICH or proportion of surgical intervention between patients prescribed aspirin and those not prescribed aspirin. 
During the follow-up period 14 incidences of recurrent ICH occurred, 11 in patients not prescribed aspirin, and three in patients prescribed aspirin. The difference was not significant (36.6 per 1,000 patient years vs. 22.7 per 1,000 patient-aspirin years, $\mathrm{p}=0.73$ ). There was also no significant difference in the incidence of ischaemic stroke (49.4 per 1,000 patient years vs. 22.3 patientaspirin-years) or acute coronary syndrome (34.7 per 1,000 patientyears vs. 17.1 per 1,000 patient-aspirin-years, $\mathrm{p}=0.53)$. When recurrent $\mathrm{ICH}$, acute coronary syndrome, and ischaemic stroke were considered together as adverse events, their incidence was significantly higher in patients not prescribed aspirin (112.8 per 1,000 patient-years, vs. 52.4 per 1,000 patient-aspirin years, $\mathrm{p}=0.04$ ). Due to the small number of patients, further analyses based on the location of ICH were not performed.

\section{Discussion}

In this study, we compared the incidence, time course and clinical predictors of recurrent $\mathrm{ICH}$, ischaemic stroke, and acute coronary syndrome in a large cohort of Chinese patients who survived a first ICH. After a mean follow-up of 62 months, $10.7 \%$ of patients had a recurrent $\mathrm{ICH}$ with a recurrence rate of 20.6 per 1,000 patient years. The incidence of recurrent ICH in patients prescribed aspirin after ICH was nonetheless similar to those not prescribed aspirin. The only predictors of recurrent ICH were advanced age and hypertension. Likewise, the incidence of ischaemic stroke and acute coronary syndrome in patients prescribed aspirin following ICH did not differ significantly to those not prescribed aspirin. Nonetheless among patients at high risk of thromboembolic events including those with coronary artery disease, ischaemic stroke, diabetes and atrial fibrillation, the use of aspirin following ICH was beneficial. It was associated with a significantly lower overall risk of recurrent $\mathrm{ICH}$, ischaemic stroke and acute coronary syndrome.

$\mathrm{ICH}$ is the most devastating and the least treatable form of stroke encountered in clinical practice. In the Caucasian population, ICH accounts only for $10-15 \%$ of all types of strokes. In stark contrast, one-third of all strokes in Chinese patients result from ICH; a three-fold higher incidence (4-6). In fact, the mean age of our study cohort appears to be 5-10 years younger than previous published non-Chinese cohorts of primary intracranial haemorrhage survivors (range from 64 to 70 years) $(8,10,18)$. Hypertension is a major risk factor for $\mathrm{ICH}$, and more importantly, the risk is related to hypertension severity and blood pressure control (19). A plausible explanation for the difference in age of onset of primary ICH could be related to the higher prevalence of hypertension, and possibly a lower awareness of hypertension in Chinese (20). Furthermore, possible synergic interactions between smoking and hypertension (given the high prevalence of cigarette smokers) (21), and a lower blood pressure threshold to ICH in Chinese (22), may also contribute to the higher incidence and younger onset of ICH in Chinese. Nonetheless, recent prospective randomised control trial has suggested that effective blood press- ure lowering treatment is likely to provide protection against all types of ICH (23). In addition to the high early mortality, patients who survive the initial phase not only suffer significant residual disability, but also face a substantial risk of recurrent ICH: the annual rate of recurrent ICH ranges from $2.1 \%$ to $3.7 \%$ in Caucasian post-ICH patients (8). Long-term data concerning recurrent $\mathrm{ICH}$ in Chinese patients has not been previously available. In the present study, the overall annual rate of recurrent $\mathrm{ICH}$ in a relative unselected cohort of Chinese patients who survived ICH was comparable with that of Caucasians (8). This similarity may be related to more aggressive control following ICH of modifiable risk factors in Chinese patients. Our study confirmed the findings of previous work that identified advanced age and hypertension as independent risk predictors for recurrent $\operatorname{ICH}(8,18,24)$.

In addition to recurrent $\mathrm{ICH}$, a previous long-term follow-up study demonstrated substantial risks among post-ICH patients for ischaemic stroke, myocardial infarction, and sudden death (8). In a cohort of 243 patients who survived ICH Vermeer et al. found that the annual rate of recurrent ICH was $2.1 \%$; the annual rate of all vascular events including recurrent $\mathrm{ICH}$, ischaemic stroke, myocardial infarction, and sudden death was as high as 5.9\% (8). This is largely because ICH and atherosclerotic disease share many risk factors. While the effectiveness of anti-platelet therapy to reduce death, ischaemic stroke and myocardial infarction has been well known in patients with established atherosclerotic disease or its equivalent, the benefits of such long-term anti-platelet therapy must be weighed against the risk of future ICH in post-ICH patients. The latter is commonly perceived to be much higher than that in patients with no history of ICH $(\sim 0.2$ events per 1,000 patient-years) (21). For example, only $44 \%$ of patients with standard indications for anti-platelet therapy were prescribed aspirin in this study, presumably related to a physicians' perceived high risk of recurrent ICH. To date, only two observational studies have addressed the issue of long-term anti-platelet therapy in post-ICH patients $(10,11)$.Viswanathan et al. studied the risk of recurrent ICH in a total of 207 post-ICH patients from a single-centre prospective cohort, in which 46 patients $(22 \%)$ were prescribed aspirin in sub-

\section{What is known about this topic?}

- Intracranial haemorrhage (ICH) accounts for a higher percentage of all strokes in Chinese than in Caucasians.

- Patients surviving the first ICH have a higher risk of recurrent ICH. As a result, anti-platelet agents and/or anticoagulants are less commonly used despite the presence of standard clinical indications.

\section{What does this paper add?}

- Chinese patients surviving ICH are at substantial risk of both recurrent ICH and ischaemic vascular events.

- The use of aspirin following ICH does not appear to increase the risk of recurrent $\mathrm{ICH}$, and among those patients with standard indications for aspirin, its use reduces the incidence of overall vascular events. 
sequent follow-up (11). After a mean follow-up of 19.5 months, 39 patients suffered recurrent ICH; use of aspirin did not appear to be associated with an increased risk of recurrent ICH (11). Likewise, a more recent study by Flynn et al. demonstrated in a cohort of 417 post-ICH patients that the use of aspirin after $\mathrm{ICH}$ was not associated with recurrent $\mathrm{ICH}$ (10).

Current international guidelines for the management of $\mathrm{ICH}$, albeit based largely on expert opinions, do not prohibit the use of aspirin in post-ICH patients with atherosclerotic disease $(25,26)$. Consistent with these two Caucasian cohorts, in the present study, the use of aspirin did not appear to have an effect the incidence in recurrent ICH. Counter-intuitively, patients prescribed aspirin after ICH appeared to have a higher incidence of ischaemic stroke. This may partly be related to their higher prevalence of atherosclerotic disease necessitating anti-platelet therapy. Many patients not prescribed aspirin after ICH had no clinical indication for antiplatelet therapy (absence of atherosclerotic disease), thus their inclusion in the analysis may mask the protective effects of anti-platelet therapy. In the subgroup analysis of patients with standard indications for anti-platelet therapy, the use of aspirin was not associated with a significantly higher incidence of ICH, or lower incidence of ischaemic stroke or acute coronary syndrome. Nonetheless, the results of the present study demonstrated that anti-platelet therapy can lower the incidence of overall vascular events (a composite of recurrent $\mathrm{ICH}$, ischaemic stroke, and acute coronary syndrome).

Cerebral microbleeds detected by gradient echo $\mathrm{T} 2{ }^{*}$ weighted MRI in patients presenting with intracerebral haemorrhage or ischaemic stroke are associated with increased risk of subsequent intracerebral haemorrhage (27). The Rotterdam scan study revealed that use of platelet aggregation inhibitors (aspirin or carbasalate calcium) was related to presence of cerebral microbleeds and cerebral microbleeds of lobar distribution were more prevalent among aspirin users than carbasalate calcium users (28). A prospective study of 104 primary lobar intracerebral haemorrhage survivors revealed that recurrence of lobar intracerebral haemorrhage was associated with previous intracerebral haemorrhage before the index event, number of lobar cerebral microbleeds and presence of CT-defined white matter hypodensity in the posterior region. In addition, subsequent aspirin use independently increased the risk of recurrent intracerebral haemorrhage (29). Detection of cerebral microbleeds in our study patients may have helped to identify those with high risk of recurrent ICH.

The results of our study have several important clinical implications. First, in addition to a substantial risk for recurrent ICH, patients who survive ICH are at risk for other thrombotic vascular events. The goal of management should thus focus more broadly on ameliorating overall cardiovascular risk and not just prevention of recurrent ICH. Initiation of anti-thrombotic therapy should be considered in all post-ICH patients with standard indications for aspirin. This is particularly important in patients at high risk of ischaemic events where the benefit of aspirin therapy may outweigh the risk of recurrent $\mathrm{ICH}$.

\section{Limitations}

This observational study had some limitations. First, the use of aspirin was determined by the individual patient's attending physician rather than by random assignment. Second, no patient was prescribed clopidogrel and/or other anti-platelet agents: our data cannot be extrapolated to patients prescribed these agents. Third, this was a hospital-based study using single-hospital registry data and did not include patients with a milder form of ICH who did not require hospitalisation. Fourth, it is well recognised that the risks of recurrent ICH vary considerably accordingly to the location of the initial ICH; for instance, lobar ICH appears to have a higher risk of recurrence than deep ICH. However, due to the small sample size and the low incidence of recurrent $\mathrm{ICH}$, we failed to perform any meaningful statistical comparison of the recurrence risk between patients with different ICH locations. Fifth, we did not utilise gradient echo $\mathrm{T}^{\star}$ weighted MRI to identify microbleeds that would be useful for risk stratification of a recurrent $\mathrm{ICH}$.

\section{Conclusion}

Patients who survive ICH are at substantial risk of both recurrent $\mathrm{ICH}$ and ischaemic vascular events. This study provides data on the long-term outcome in post-ICH Chinese patients that may allow better risk stratification in such patients. The use of aspirin following ICH does not appear to increase the risk of recurrent $\mathrm{ICH}$, but in those patients with standard indications for aspirin, its use reduces the incidence of overall vascular events.

\section{Conflict of interest}

None declared.

\section{References}

1. Feigin VL, Lawes CM, Bennett DA, et al. Stroke epidemiology: a review of population-based studies of incidence, prevalence, and case-fatality in the late 20th century. Lancet Neurol 2003; 2: 43-53.

2. Kolominsky-Rabas PL, Sarti C, Heuschmann PU, et al. A prospective communitybased study of stroke in Germany--the Erlangen Stroke Project (ESPro): incidence and case fatality at 1, 3, and 12 months. Stroke 1998; 29: 2501-2506.

3. Thrift AG, Dewey HM, Macdonell RA, et al. Incidence of the major stroke subtypes: initial findings from the North East Melbourne stroke incidence study (NEMESIS). Stroke 2001; 32: 1732-1738.

4. Yang QD, Niu Q, Zhou YH, et al. Incidence of cerebral hemorrhage in the Changsha community. A prospective study from 1986 to 2000. Cerebrovasc Dis 2004; 17: 303-313.

5. Zhang LF, Yang J, Hong Z, et al. Proportion of different subtypes of stroke in China. Stroke 2003; 34: 2091-2096.

6. Huang CY, Chan FL, Yu YL, et al. Cerebrovascular disease in Hong Kong Chinese. Stroke 1990; 21: 230-235.

7. Broderick J, Connolly S, Feldmann E, et al. Guidelines for the management of spontaneous intracerebral hemorrhage in adults: 2007 update: a guideline from the American Heart Association/American Stroke Association Stroke Council, High Blood Pressure Research Council, and the Quality of Care and Outcomes in Research Interdisciplinary Working Group. Stroke 2007; 38: 2001-2023. 
8. Vermeer SE, Algra A, Franke CL, et al. Long-term prognosis after recovery from primary intracerebral hemorrhage. Neurology 2002; 59: 205-209.

9. Wani M, Nga E, Navaratnasingham R. Should a patient with primary intracerebral haemorrhage receive antiplatelet or anticoagulant therapy? Br Med J 2005; 331: 439-442.

10. Flynn RW, MacDonald TM, Murray GD, et al. Prescribing antiplatelet medicine and subsequent events after intracerebral hemorrhage. Stroke 2010; 41: 2606-2611.

11. Viswanathan A, Rakich SM, Engel C, et al. Antiplatelet use after intracerebral hemorrhage. Neurology 2006; 66: 206-209.

12. Ho LY, Siu CW, Yue WS, et al. Safety and efficacy of oral anticoagulation therapy in Chinese patients with concomitant atrial fibrillation and hypertension. J Hum Hypertens 2011; 25: 304-310.

13. Siu CW, Jim MH, Ho HH, et al. Transient atrial fibrillation complicating acute inferior myocardial infarction: implications for future risk of ischemic stroke. Chest 2007; 132: 44-49.

14. Siu CW, Jim MH, Lau CP, et al. Low molecular weight heparin versus unfractionated heparin for thromboprophylaxis in patients with acute atrial fibrillation: A randomized control trial. Acute Card Care 2011; 13: 196-198.

15. Siu CW, Pong V, Jim MH, et al. Beta-blocker in post-myocardial infarct survivors with preserved left ventricular systolic function. Pacing Clin Electrophysiol 2010; 33: 675-680.

16. Ho HH, Kwok OH, Jim MH, et al. Long-term clinical outcomes after intravascular brachytherapy for instent restenosis and de novo coronary artery lesions in percutaneous coronary intervention. Cardiovasc Revasc Med 2011; 12: 152-157.

17. Ho HH, Pong V, Siu CW, et al. Long-term clinical outcomes of drug-eluting stents vs bare-metal stents in Chinese patients. Clin Cardiol 2010; 33: E22-29.

18. Passero S, Burgalassi L, D'Andrea P, et al. Recurrence of bleeding in patients with primary intracerebral hemorrhage. Stroke 1995; 26: 1189-1192.

19. Hart RG, Tonarelli SB, Pearce LA. Avoiding central nervous system bleeding during antithrombotic therapy: recent data and ideas. Stroke 2005; 36: 1588-1593.
20. Xu T, Wang Y, Li W, et al. Survey of prevalence, awareness, treatment, and control of hypertension among Chinese governmental and institutional employees in Beijing. Clin Cardiol 2010; 33: E66-72.

21. Nakamura K, Barzi F, Lam TH, et al. Cigarette smoking, systolic blood pressure, and cardiovascular diseases in the Asia-Pacific region. Stroke 2008; 39: 1694-1702.

22. Zhang XF, Attia J, D'Este C, et al. The relationship between higher blood pressure and ischaemic, haemorrhagic stroke among Chinese and Caucasians: metaanalysis. Eur J Cardiovasc Prev Rehabil 2006; 13: 429-437.

23. Arima H, Tzourio C, Anderson C, et al. Effects of perindopril-based lowering of blood pressure on intracerebral hemorrhage related to amyloid angiopathy: the PROGRESS trial. Stroke 2010; 41: 394-396.

24. Bae $\mathrm{H}$, Jeong $\mathrm{D}$, Doh J, et al. Recurrence of bleeding in patients with hypertensive intracerebral hemorrhage. Cerebrovasc Dis 1999; 9: 102-108.

25. Morgenstern LB, Hemphill JC, 3rd, Anderson C, et al. Guidelines for the management of spontaneous intracerebral hemorrhage: a guideline for healthcare professionals from the American Heart Association/American Stroke Association. Stroke 2010; 41: 2108-2129.

26. Steiner T, Kaste M, Forsting M, et al. Recommendations for the management of intracranial haemorrhage - part I: spontaneous intracerebral haemorrhage. The European Stroke Initiative Writing Committee and the Writing Committee for the EUSI Executive Committee. Cerebrovasc Dis 2006; 22: 294-316.

27. Lovelock CE, Cordonnier C, Naka H, et al. Antithrombotic drug use, cerebral microbleeds, and intracerebral hemorrhage: a systematic review of published and unpublished studies. Stroke 2010; 41: 1222-1228.

28. Pang GF, Cao YZ, Fan CL, et al. Determination of clopidol residues in chicken tissues by liquid chromatography: collaborative study. J AOAC Int 2003; 86 : 685-693.

29. Biffi A, Halpin A, Towfighi A, et al. Aspirin and recurrent intracerebral hemorrhage in cerebral amyloid angiopathy. Neurology 2010; 75: 693-698. 